\title{
ERRATUM
}

\section{Connexin mutations associated with palmoplantar keratoderma and profound deafness in a single family}

David P Kelsell, Amanda L Wilgoss, Gabriela Richard, Howard P Stevens, Colin S Munro and Irene M Leigh

Figure 1 in the above paper was printed incorrectly. The complete paper, with the correct Figure 1, has been reproduced in full on the following pages. The online version of the paper has been corrected in the original. 